\title{
LA JUNTA PARA AMPLIACIÓN DE ESTUDIOS Y EL DESARROLLO DE LA PSICOLOGÍA ESPAÑOLA
}

\author{
Helio Carpintero y Fania Herrero
}

Universidad Complutense de Madrid; UNED

\begin{abstract}
RESUMEN
Durante las últimas décadas del s. XIX una serie de movimientos culturales y reformistas iniciaron la renovación de la ciencia y la cultura españolas, que habían de tener sus cimientos asentados en el proceso de la educación, llave de ulteriores transformaciones en el campo del desarrollo tecnológico, científico y social. Con un retraso considerable nuestro país comenzó un proceso que se había vivido en otros países occidentales, y ponía así la proa hacia una europeización de la sociedad española. Uno de los logros de esta obra renovadora fue la Junta para Ampliación de Estudios e Investigaciones Científicas (JAE), muy especialmente a través de su política de becas (19071936). En el presente trabajo presentamos un recorrido por las instituciones y las figuras que, a través de la JAE, hicieron de puente entre la psicología europea y la española, siendo responsables de que en sólo tres décadas esta ciencia alcanzara en nuestro país el nivel científico propio de un país desarrollado. Cabe destacar el papel jugado en esta historia por la Escuela de Ginebra, cuyas relaciones con los profesionales españoles, propiciadas por la Junta, iban a determinar el desarrollo posterior de la psicología en nuestro país.
\end{abstract}

PALABRAS CLAVE: Junta para Ampliación de Estudios, Historia de la Psicología en España, Instituto J.J. Rousseau.

\section{LA JUNTA PARA AMPLIACIÓN DE ESTUDIOS AND THE DEVELOPMENT OF SPANISH PSYCHOLOGY}

\begin{abstract}
During the last decades of the XIXth. century, there was an awakening of consciousnesss for the need of a Spanish cultural renovation, of which one of the aims was to create and develop a Spanish science, resembling the scientific models already established in more advanced countries. There was a desire of europeanization. Since it was a global social objective, it was necessary to start from the educator's training. In this climate the Junta para Ampliación de Estudios e Investigaciones Científicas, appeared. The role that the Junta played in Spanish research and in the innovation in the Psychopedagogical field through the first third of the XXth. century was extremely important. The Junta's policy of scholarships was one of its most substantial achievements, for it made possible that the country reached in a few decades (1907-1936) the European scientific and
\end{abstract}


psychological level. The relations among Spanish teachers and the Institute J.J. Rousseau is to be highlighted, as «Geneva School» was to influence deeply the further development of psychology in Spain.

KEY WORDS: Junta para Ampliación de Estudios, History of Psychology in Spain, Institute J.J. Rousseau.

Durante las últimas décadas del s. XIX una serie de movimientos culturales y reformistas iniciaron la renovación de la ciencia y la cultura españolas, liberándolas de los conflictos ideológicos que impedían su avance. Acompañaba a este objetivo general un deseo de reformas sociales de carácter global, que habían de tener sus cimientos asentados en el proceso de la educación, llave de ulteriores transformaciones en el campo del desarrollo tecnológico, científico y social. Con un retraso considerable, al menos de una generación, nuestro país comenzó un proceso que se había vivido en otros países occidentales ya en las décadas anteriores, y ponía así la proa hacia una europeización de la sociedad española.

En este entorno hay que destacar la obra realizada por profesores y científicos relacionados, de un modo más o menos inmediato, con la Institución Libre de Enseñanza (ILE). Esta obra, fundada por Francisco Giner de los Ríos en 1876, vino a ser la principal impulsora de la reforma educativa española, que partiría no sólo de una modernización de los contenidos de la enseñanza sino, lo que era aún más importante, de la actualización y renovación de los conocimientos y métodos de los propios educadores.

Uno de los logros de esta obra renovadora fue la Junta para Ampliación de Estudios e Investigaciones Científicas (JAE) (1907), institución cuya importancia ha sido ampliamente reconocida y, en las últimas décadas, ha merecido la atención de numerosos estudios sobre su papel en el desarrollo de las diferentes áreas de conocimiento ${ }^{1}$. En el presente trabajo, inscrito como muchos

1 Gómez Orfanell, G. (1976), «La Junta para Ampliación de Estudios y la política de pensiones en el extranjero». Revista de Educación, 243; LAPORTA, F. et al. (1980), La Junta para Ampliación de Estudios e Investigaciones Cientificas, Madrid, Fundación Juan March; MARín, T. (1986), «Los becados de la JAE y la Pedagogía alemana». Revista de Educación, 280; MARín, T., «Modelo educativo de los becados por la J.A.E.». En SÁNCHEZ Ron, J. (coord.) (1988), La Junta para Ampliación de Estudios e Investigaciones Científicas, ochenta años después, 2 vols., Madrid, CSIC; MARÍN, T. (1990), La renovación pedagógica en España (1907-1936). Los Pensionados en pedagogía por la Junta para Ampliación de Estudios, Madrid, CSIC; LAPORTA, F., et al. (1987), «La Junta para Ampliación de Estudios I, II», Arbor, 493, pp. 499-500; MORENO, A. y SÁNCHEZ Ron, J.M. (1987), «La JAE: La vida breve 
otros en el marco de la conmemoración de su centenario, presentamos un recorrido por las instituciones y las figuras que, a través de la JAE, hicieron de puente entre la psicología europea y la española, siendo responsables de que en sólo tres décadas esta ciencia alcanzara en nuestro país el nivel científico propio de un país desarrollado.

\section{CREACIÓN Y EVOLUCIÓN DE LA JAE}

A efecto de simple recordatorio, tengamos presente que la Junta para Ampliación de Estudios e Investigaciones Científicas se creó por Real Decreto del 11 de enero de 1907. Su primer Presidente fue, durante largos años, Santiago Ramón y Cajal (1852-1934), galardonado el año anterior con el premio Nobel, quien representaba un símbolo vivo de las posibilidades reales de una ciencia nacional. El eminente profesor de Histología, entonces ya con reputación mundial por sus investigaciones sobre el sistema nervioso, aceptó aquella responsabilidad como parte de su esfuerzo personal a favor de la regeneración científica del país. La Junta contó también con la labor extraordinaria de quien fue su Secretario Permanente, a lo largo de 30 años, José Castillejo (1877-1945), que hizo posible el complejo funcionamiento de la nueva organización. Se nombró también como vocales ${ }^{2}$ a una serie de personalidades y científicos eminentes que representaban las diferentes ramas del conocimiento y los variados matices del espectro de la opinión pública. Merecen ser recordados los primeros consejeros, que encauzaron el curso de la obra poste-

de una fundación ahora octogenaria», Mundo Científico, 65; CARPINTERO, H., «La Psicología y la JAE. Una primera aproximación». En SÁNCHEZ Ron, J.M. (1988); GARCíA, E., y HeRRERO, F. (1994), «La Psicología y los pensionados en psicología por la Junta para Ampliación de Estudios. Análisis cuantitativo», Revista de Historia de la Psicología, 15 (3-4), pp. 251-265; García, E., y Herrero, F. (1995), «Neuropsiquiatría y Psicología en España en el primer tercio del s. XX. El papel de la JAE». En VV.AA., Un siglo de Psiquiatría en España, Madrid, Extra Editorial, pp. 111-140; GARCíA, E., y HERrero, F. (1996), «Psicología y Educación en la España de Preguerra. La influencia de J. Piaget y el Instituto J.J. Rousseau», Revista de Historia de la Psicología, 17 (3-4), pp. 166-176; Herrero, F., GArCía, E., y CARPINTERo, H. (1995), «Psicopedagogía en España (1900-1936). Becarios españoles en centros europeos», Revista de Historia de la Psicología, 16 (1-2), pp. 181-200; PESET, J.C. y HERNÁNDEZ SANDOICA, E., «Instituciones científicas y educativas». En JOVER, S.M. (dir.) (1994), Historia de España Menéndez Pidal, 39. Madrid, Espasa Calpe; etc.

2 Junta Para Ampliación de Estudios e InVestigaciones Científicas (1907-1934), Memorias, Madrid. 
rior: José Echegaray, Marcelino Menéndez Pelayo, Joaquín Sorolla, Joaquín Costa, Vicente Santamaría de Paredes, Alejandro San Martín, Julián Calleja y Sánchez, Eduardo Vincenti, Gumersindo de Azcárate, Luis Simarro, Ignacio Bolívar, Ramón Menéndez Pidal, José Casares Gil, Adolfo Álvarez Buylla, José Rodríguez Carracido, Julián Ribera Tarragó, Leonardo Torres Quevedo, José Marvá, José Fernández Jiménez y Victoriano Fernández Ascarza.

Da perfecta idea de los objetivos de la institución la declaración que de ellos se hace en el artículo $1^{\circ}$ de sus Estatutos. En efecto, allí se indica que entre sus propósitos se incluía el establecimiento de: «a) El Servicio de ampliación de estudios dentro y fuera de España. b) Las delegaciones en congresos científicos. c) El servicio de información extranjera y relaciones internacionales en materia de enseñanza. d) El fomento de trabajos de investigación científica. e) La protección de las instituciones educativas en la enseñanza secundaria y superior» ${ }^{3}$.

Tras algunas dificultades iniciales en su andadura, y afectada en ocasiones por los vaivenes de la política, la JAE iría cumpliendo uno por uno, aunque con diferente intensidad, sus varios objetivos. La creación de centros de investigación como el Centro de Estudios Históricos y el Instituto Nacional de Ciencias Físico-Naturales con todos sus laboratorios, y centros educativos como la Residencia de Estudiantes, renovó el horizonte cultural del país. En 1918 se crea el Instituto Escuela como espacio de experimentación e innovación pedagógica. La dictadura de Primo de Rivera introdujo limitaciones y cortapisas, pero permitió el fortalecimiento de las relaciones con la Fundación Rockefeller en materia de sanidad y educación ${ }^{4}$. Durante la Segunda República (1931-1936) fue también compleja la vida de la JAE, dados los avatares de la política educativa, pero se obtuvieron nuevos logros como la incorporación del edificio donado por la Fundación Rockefeller para el Instituto Nacional de Física y Química, lo que permitió una notable mejora en la capacidad investigadora. Y así llegamos a la Guerra Civil, periodo durante el cual es difícil seguir la vida de la JAE (la Memoria de los cursos 1935-36 no llegó a redactarse. José Castillejo, probable redactor de las Memorias, había salido hacia Inglaterra en el verano del 36, amenazado de muerte). La sede de la JAE, en Madrid (Medinaceli, 4), estaba en poder de los milicianos, y la mayoría de sus edificios se utilizaron para necesidades militares. En 1936 una orden de 11 de septiembre declaró caducadas todas las pensiones. A lo largo de la gue-

3 Gaceta (1910, 28 de enero). Real Decreto de 22 de enero de 1910, modificando el Real Decreto constitutivo de la JAE.

4 LAPORTA, F., et al. (1987). 
rra la Junta presta su apoyo al gobierno republicano. Por otro lado, en la llamada «zona nacional» se crea a comienzos de 1938 el Ministerio de Educación Nacional que disuelve la JAE (19 de mayo de 1938) y crea en su lugar el Consejo Superior de Investigaciones Científicas (noviembre, 1939), que pone fin a toda la línea educativa precedente 5 .

En conjunto, su obra fue muy considerable. Entre 1907 y 1934 la Junta concedió 1594 becas de estancia en el extranjero, individuales y en grupo, de las que más del $50 \%$ correspondieron a maestros y profesores para estudios pedagógicos, y otro $38 \%$ a médicos ${ }^{6}$. Ello respondía al general reconocimiento de unas ciertas prioridades: la necesidad de una reforma pedagógica, por un lado, y por otro, de la sanidad pública.

\section{LA PSICOLOGÍA Y LAS PENSIONES DE LA JUNTA}

La Institución Libre de Enseñanza tuvo muy pronto un interés marcado por la nueva psicología. Veía en ella uno de los pilares científicos sobre los que había de basarse una ciencia moderna de la educación. Esta había de tener en cuenta las aptitudes y motivaciones que están en juego en el complejo proceso educativo. Don Francisco Giner mismo había escrito unas Lecciones sumarias de psicología (1874), con la colaboración de Eduardo Soler y Alfredo Calderón, libro que, a pesar de su destino escolar, mereció la atención y los elogios de distinguidos especialistas de la época. Y unos años más tarde, en el Museo Pedagógico Nacional (1882) dirigido por Manuel B. Cossío, se estableció un laboratorio (1893) dirigido durante un tiempo por Luis Simarro, figura ligada inicialmente a la Institución y primer catedrático de psicología en la Universidad de Madrid.

Estos hombres buscaron conciliar la filosofía krausista, que inspiró su movimiento en sus orígenes, con la nueva ciencia de la mente que el estructuralismo de W. Wundt, y el funcionalismo de W. James o J. Dewey, habían situado en el campo del saber empírico acerca del hombre.

El interés por estos temas resulta evidente en las páginas del Boletín de la Institución Libre de Enseñanza (BILE), donde se fueron ofreciendo trabajos de autores españoles - J. Sanz del Río, L. Simarro, J. Caso, M. Navarro, D. Barnés, J. Mallart y tantos más- junto con los debidos a plumas extranjeras

5 SÁNCHEZ RON (coord.) (1988).

6 Herrero, García y CARPintero (1995). 
- Stanley Hall, Binet, Claparède, Wallon... — , en un esfuerzo por ampliar el horizonte intelectual del mundo cultivado de la época.

Otro efecto de esa preocupación por la psicología es, sin duda, el sólido apoyo que la JAE iba a prestar a jóvenes educadores e investigadores interesados en temas psicológicos, a los que se concedieron ayudas y pensiones para viajes o estancias en centros extranjeros. Ciertamente, la psicología atrajo a muchos que luego iban a dedicarse a otros campos del saber. La proximidad con todo lo humano que siempre ha tenido aquella ciencia ha invitado a muchos investigadores e intelectuales curiosos a incorporarla, al menos en sus líneas generales, a su bagaje cultural como complemento de otros temas de su especial preocupación. Por eso no es de extrañar que, a la hora de diseñar programas de trabajo, muchos pensionados procedentes del mundo médico, jurídico y humanístico incluyeran alguna materia psicológica entre sus opciones de estudio.

Hemos de partir del hecho de que en esa época no había ningún titulado en psicología, esto es, no había propiamente ningún psicólogo, al no haber estudios reglados de esa especialidad. Había, en cambio, una asignatura de psicología en la enseñanza media, dentro del conjunto de materias filosóficas, que se había introducido a mediados del siglo anterior (plan de Gil de Zárate, de 1845). Esto hizo que se interesaran por ella los profesores de bachillerato, primero en un sentido filosófico y explícitamente 'espiritualista', como en el plan de estudios del Ministro García Alix, de 1900, y luego ya claramente modernizado y orientado hacia la psicología empírica en el 'plan Callejo' de 19277. Los manuales escritos por aquellos profesores van dando testimonio de los avatares de esta enseñanza, y también del creciente interés personal hacia la nueva ciencia que se fue despertando en muchos de ellos.

De esta suerte, los educadores, preocupados por la enseñanza en la escuela; los profesores de bachillerato, encargados de enseñar esa materia a sus alumnos; los médicos y educadores interesados en la enseñanza terapéutica y la educación de niños con problemas orgánicos y mentales; los abogados y juristas preocupados por la personalidad de los delincuentes y la naciente criminología, todos estos grupos vinieron a interesarse por la nueva psicología como complemento útil a su formación especializada.

Por ello, hemos creído de interés considerar incluidos en nuestro grupo todos aquellos pensionados cuyo tema estaba relacionado claramente con los campos de estudio de la psicología, al haber elegido alguna materia de esa especialidad en sus peticiones. Comprobamos así que una serie de médicos, de educadores y

7 FEY, E. (1975). Estudio documental de la filosofía en el bachillerato español (18071957), Madrid, CSIC, p. 59. 
filósofos, como acabamos de explicar, se interesó por visitar y conocer los cursos y enseñanzas relacionados con la nueva psicología que se estaba haciendo en algunos países de nuestro entorno cultural. Por el contrario, no hemos recogido a aquellas figuras cuya obra ha sido al cabo significativa o relevante para el desarrollo de la historia de la psicología española, como hemos hecho en otros trabajos ya mencionados, ni a aquellos que, habiendo estudiado temas de campos afines a la psicología (orientación profesional, psiquiatría, etc.), no realizaron estudios específicamente psicológicos durante sus viajes.

De este modo, hemos estudiado un total de 122 pensiones concedidas a 102 pensionados, a los que hemos añadido la información disponible sobre dos figuras importantes de la psicología española pensionadas durante los periodos en los que no se publicaron las Memorias de la JAE (J. Jaén y J. Peinado). Ciertamente, no todas las pensiones fueron iguales. Si bien la mayoría de ellas duraron entre 3 y 12 meses, visitando cada pensionado una media de 2,1 países durante su beca, también hay otras que duraron unos pocos días, por ejemplo aquellas dadas para asistir a congresos, donde empezó a ser normal la presencia española. En todos los casos, las ayudas de la JAE representaron un poderoso instrumento de apertura y conocimiento de la ciencia internacional, de que el país se hallaba necesitados.

\section{Pensionados con cursos de psicología}

A partir de los datos de las Memorias publicadas, se ha podido confeccionar la lista de pensionados de la JAE que cursaron estudios de Psicología, en alguna - o varias - de sus especialidades. Como se ha dicho, en algunos casos estos becarios disfrutaron de más de una beca, por lo que es dispar el número de pensiones y el de pensionados. El listado completo se encuentra en el Anexo 1.

Se recogen aquí quienes estudiaron temas psicológicos durante sus pensiones: psicología general, experimental, animal, diferencial, evolutiva, de la afectividad, del pensamiento, del lenguaje, del trabajo, etc., aunque pudieron también hacer cursos de otras temáticas diferentes. Entre ellos se encuentran especialistas de distinta formación y profesión. Los agrupamos en tres distintas categorías: 1) la de aquellos que son normalmente incluidos en la trama de la historia de la psicología española ${ }^{8}$; 2) el colectivo de educadores - maestros, inspectores y profesores de Escuelas Normales-, el más ampliamente repre-

8 CArpintero, H. (2004), Historia de la Psicología en España. Madrid, Pirámide; SÁIz, D. y SÁiz, M. (eds.) (1996). Personajes para una Historia de la Psicología en España, Barcelona, Pirámide. 
sentado aquí; 3) un tercer nivel que incluye a 'otros profesionales', y que reúne a juristas, filósofos, profesores de bachillerato y médicos, los cuales eligieron seguir alguno o varios cursos psicológicos.

Probablemente el interés por el movimiento europeo de la Escuela Nueva -Pestalozzi, Froebel, etc.- - y la mayor difusión del conocimiento del francés entre los estudiantes de la época, expliquen en gran parte el que Francia, Bélgica y Suiza fueran los países más visitados, aunque los pensionados en filosofía y en temas neuropsiquiátricos prefirieron por lo general Alemania.

1) Especialistas estrechamente relacionados con la psicología.

Hay un grupo de nombres claramente vinculados, temporal o plenamente, a la psicología, que recibieron el apoyo y el estímulo de la JAE. Incluimos aquí, ordenados alfabéticamente, a: Julián Besteiro, Juan Comas, Federico Dalmau, Ángel Garma, José Germain, Fermín Herrero, Juan Jaén, Eloy Luis André, Roberto Nóvoa, José Peinado, Santiago Ramón y Cajal, Mercedes Rodrigo, Cipriano Rodrigo Lavín, Gonzalo Rodríguez Lafora, Juan Roura, Luis Simarro y Juan Vicente Viqueira.

Es un grupo complejo. Hay en él individuos procedentes al menos de cuatro generaciones, con formaciones distintas, con especialización diversa, pero todos, en una u otra forma, han contribuido con su trabajo y sus ideas a incrementar el torso de la psicología española.

Hay dos figuras que son las de más edad del grupo, que habría que situar en la generación de 1856 — generación de 'sabios' —, verdaderos pioneros de todo este campo: Luis Simarro Lacabra (n.1851), psiquiatra y catedrático de psicología experimental en la Universidad de Madrid (1902), y Santiago Ramón y Cajal (n.1852), el genial descubridor de la neurona y Premio Nobel de Medicina en 1906. Los dos fueron miembros del Patronato de la Junta, y sin duda recibieron apoyo para ampliar contactos y relaciones con otros grupos extranjeros. Los dos, cada uno en su línea, iban a impulsar el estudio de la psicología y de sus bases psicobiológicas, y además dejarían tras de sí una cola de discípulos que iban a continuar el proceso abierto por ellos en línea con la europeización del país. Se ha de notar que muchos de los discípulos más directos de Cajal aparecen disfrutando de pensiones cuya temática es la neurología o fisiología, no la psicología, (P. del Río Hortega, N. Achúcarro, etc.), razón por la que quedan fuera de nuestro campo temático.

Hallamos luego cuatro figuras, pertenecientes ya a la generación 'del 98' (o generación de 1871, en la serie propuesta reiteradamente por J. Marías - 
Marías, 1960): Julián Besteiro (n.1870), Fermín Herrero (n.1871) Eloy Luis André (n.1876) y Federico Dalmau Gratacós (n.1874). Todos estos nombres tienen un rasgo común, y es su interés por una psicología de raíz wundtiana, orientada al estudio de las sensaciones y la psicofísica. Todos son profesores de filosofía de instituto de enseñanza media - aunque Besteiro pasará pronto (1914) a la cátedra de Lógica de la Universidad de Madrid (Bandrés y Llavona, 1991) ${ }^{10}$ - y han escrito libros de texto de psicología para su uso en las clases de bachillerato. Herrero y Luis André seguirán muy de cerca los textos wundtianos ${ }^{11}$, y Dalmau se orienta hacia la neoescolástica.

Julián Besteiro Fernández, (Madrid, 1870- Carmona, 1940), catedrático de filosofía de instituto y luego de lógica de la Universidad de Madrid (1912), fue una figura notabilísima del socialismo, y autor de un estudio sobre $L a$ psicofísica (1897), obra precursora de los trabajos sobre psicología científica en nuestro país; obtuvo una pensión (1909-1911) durante la que estudió con T. Lipps en Munich y con P. Barth en Leipzig, realizando en ese tiempo la traducción de una obra de Kant, los Prolegómenos a toda metafísica del porvenir (Lamo, 1973) ${ }^{12}$. Luego derivó hacia otros campos filosóficos.

Eloy Luis André (Mourazos, Orense, 1876-Madrid, 1935), catedrático en el Instituto San Isidro de Madrid, autor de libros de texto y traductor de Wundt ${ }^{13}$, por su parte tradujo y editó obras del gran psicólogo alemán con amplios prólogos dedicados al estudio de su obra y de las mentalidades nacionales, en especial la de Alemania y España ${ }^{14}$, así como el sentimiento regional de su Galicia natal. Publicó un interesante manual de psicología experimental.

Federico Dalmau y Gratacós (Bañolas, Gerona, 1874-Gerona, 1926), catedrático de filosofía del instituto de Gerona, y sacerdote, fue autor de alguna monografía sobre psicología (La sensación, 1907). Amplió estudios sobre

9 Marías, J. (1960), Ortega I. Circunstancia y vocación, Madrid, Revista de Occidente.

10 BAndRÉs, J. y LlavonA, R. (1991), «Julián Besteiro y la introducción de la psicología científica en España», Revista de Historia de la Psicología, 12 (3-4), pp. 259-268.

11 ARIAS, F.J. (1999), «Herrero Bahíllo y su psicología de la raza latina», Revista de Historia de la Psicología, 20 (3-4), pp. 291-296.

12 LAmo De Espinosa, E. (1973), Filosofia y política en Julián Besteiro, Madrid, Cuadernos para el Diálogo.

13 Carpintero, H. (2004); De Castro, R., Castro, J. y Sánchez, R. (1993), «Una aproximación biográfica a la figura de Eloy Luis André (1876-1935) desde la Historia de la Psicología», Revista de Historia de la Psicología, 14 (3-4), pp. 515-524.

14 De Castro, R., CAStro, J. y SÁnChez, R. (1993). 
psicología experimental en Lovaina con el apoyo de la JAE. Es la suya una psicología de orientación neoescolástica ${ }^{15}$.

Finalmente, mencionaremos a Fermín Herrero Bahíllo (Palencia, 1874Ávila, 1921) catedrático de los institutos de Lérida y Ávila, autor de un excelente manual construido desde la psicología de Wundt, y de trabajos sobre psicología de la religión y de las mentalidades ${ }^{16}$.

La generación de 1886 reúne a varias figuras médicas y a dos nombres que tienen ya un inconfundible perfil de psicólogo. Son los primeros Gonzalo Rodríguez Lafora (n.1886), Roberto Nóvoa Santos (n.1885), y Cipriano Rodrigo Lavín (n.1881); a ellos se suman dos figuras cuya obra y cuya actuación ha sido la propia de especialistas en temas psicológicos: Juan Vicente Viqueira (n.1886) y Mercedes Rodrigo Bellido (n.1891), la primera y única mujer de este grupo. Aquí aparecen nuevos aires, tanto en la teoría como en la práctica. Por lo pronto, hay un interés por la psicología en sus aplicaciones al mundo clínico y educativo.

Gonzalo Rodríguez Lafora (Madrid, 1886-1971) fue una gran figura de la psiquiatría de su tiempo. En el campo psicológico, trabajó en USA con S.I. Franz en problemas de cerebro y aprendizaje, luego investigaría sobre localizaciones cerebrales y sobre el problema del sueño. Fundó, con Ortega y Sacristán, los Archivos de Neurobiología (1920). Trabajó un tiempo breve en el Patronato Nacional de Anormales, y publicó un manual sobre Los niños mentalmente anormales (191717), creando además un Instituto Médico Pedagógico (1925), donde trabajarán con él José Germain y Mercedes Rodrigo ${ }^{18}$. Se interesó por el estudio de la personalidad ${ }^{19}$, y realizó una importante obra de divulgación en la prensa ( $E l \mathrm{Sol}$ ). Presidió el Consejo Superior Psiquiátrico, en los años de la república, y tras la guerra se exilió a México, de donde regresó en 1947 a España ${ }^{20}$.

15 Llavona, R. y BAndrÉs, J. (1999), «Federico Dalmau y Gratacós (1874-1926): Psicología experimental y neoescolástica española», Revista de Historia de la Psicología, 20 (34), pp. 159-166.

16 ARIAS, F.J. (2000), «Fermín Herrero Bahíllo y su investigación sobre la psicología de la religión», Revista de Historia de la Psicología, 21(2-3), pp. 65-71.

17 RODRÍGUEZ LAFORA, G. (1917), Los niños mentalmente anormales, Madrid, La Lectura.

18 Valenciano, L. (1977), El Doctor Lafora y su época, Madrid, Morata.

19 Carpintero, H., Mestre, M.V. y DEL Barrio, M.V. «Lafora y el concepto de personalidad». En Rosa, A., QuintanA, J. y LAfuente, E. (eds.) (1988), Psicología e Historia, Madrid, Eds. U. Autónoma.

20 VV.AA. (1982), El exilio español en México, México D.F., Fondo de Cultura Económica. 
Roberto Novoa Santos (Coruña, 1885-Santiago de Compostela, 1933) fue figura sobresaliente de la medicina de su tiempo, catedrático de patología de Santiago y de Madrid, y en su libro Cuerpo y espíritu (1930) desarrolló una personal doctrina energética del psiquismo, no exenta de derivaciones parapsicológicas. Se interesó por la psicología objetiva y evolucionista, y por las entonces innovadoras ideas psicoanalíticas ${ }^{21}$. Finalmente, con Novoa «la psicopatología deberá entrar definitivamente en la fisiopatología»22. Se trata, pues, de una psicología que se integra en la praxis, y no queda reducida al nivel de una mera disciplina, y disciplina de bachillerato, como hicieran los hombres de la generación anterior.

Cipriano Rodrigo Lavín (S. Sebastián, 1881-Madrid, 1972), médico, colaborador de Simarro en la cátedra de Psicología experimental de la Universidad de Madrid desde 1911, y con una larga historia de pensión de la JAE, que terminó realizando en Inglaterra, donde trabajó con Bartlett en Cambridge en 1920; aspiró sin éxito a suceder a Simarro en la cátedra madrileña, publicó algunos trabajos de psicología experimental, y luego, tras la guerra, quedó separado de la actividad universitaria, dejando un considerable número de trabajos inéditos ${ }^{23}$.

Juan Vicente Viqueira López (Madrid, 1886-Bergondo, Coruña, 1924), formado en la ILE y, más tarde, con estudios en varios centros europeos, fue catedrático de instituto en La Coruña y figura muy destacada de la psicología ${ }^{24}$. tuvo también un papel destacado en la importación de las ideas filosóficas y psicológicas alemanas a nuestro país. Disfrutó de una beca de dos años, durante los que estudiaría fundamentalmente psicología alemana actual y psicofísica en las universidades de Berlín, Leipzig y Gottinga con Rupp, Wundt y G.E. Müller, entre otros. Publica un verdadero trabajo de investigación experimental sobre memoria en una revista alemana, en 1916, resultado de sus estudios con Müller, seguido más tarde de un breve texto sobre psicología educativa. Se publicó, póstumamente, su libro sobre La psicología contemporánea (1930), excelente historia de la psicología fuertemente orientada hacia el mundo europeo.

21 Fernández Teijeiro, J.J. (2001). Más allá de la patología. La psicología de Nóvoa Santos, Santiago, Universidade de Santiago de Compostela.

22 id., p. 35.

23 QUINTANA, J. (2003), «Una aproximación a la biografía intelectual y profesional del Dr. Rodrigo Lavín, docente en la cátedra de Psicología Experimental de la Facultad de Ciencias de la Universidad Central», Revista de Historia de la Psicología, 24 (3-4), pp. 441-456.

24 Mestre, V. y CARPintero, H. (1982), «Psicólogos españoles: Juan V. Viqueira López (1886-1924)», Revista de Historia de la Psicología, 2(1), pp. 37-55; BlanCO, F., «J.V. Viqueira». En SÁIz, D. y SÁIz, M. (eds.) (1996), pp. 269-282; CARPINTERO, H. (2004). 
Completa este grupo Mercedes Rodrigo Bellido (Madrid, 1891-Puerto Rico, 1982). Es la primera mujer que cabe considerar en España como psicóloga, pues tras formarse como maestra, se especializó en Ginebra, colaborando con Piaget y formándose en psicotecnia, trabajando en tests, y adquiriendo una formación práctica. Trabajó en el Instituto Médico-Pedagógico de Lafora, y luego en el Instituto Nacional de Psicología Aplicada de Madrid, del que fue directora durante los años de la guerra civil. Exiliada en Colombia, fundó allí la carrera de psicología, pero, expulsada de nuevo, terminó sus días como psicóloga en Puerto Rico ${ }^{25}$.

Finalmente, veamos el núcleo representado por la generación de 1901. Incluimos aquí a Juan Comas (n.1900), Ángel Garma (n.1904), José Germain (n.1897), Juan Roura (n.1897), Juan Jaén (n.1904) y —añadiremos al grupo por su precoz actividad y su vinculación estrecha a Jaén- José Peinado (n.1909). Aquí encontramos nuevas influencias operando sobre la interpretación que se tenga de la psicología, especialmente el psicoanálisis, y el interés por la especialización y la praxis psicológica.

El psicoanálisis orienta de modo decisivo la obra y la posición personal de Ángel Garma Zubizarreta (Bilbao, 1904-Buenos Aires, 1993). Médico, discípulo de Marañón, se psicoanaliza en Viena con T. Reik, siendo el primer psicoanalista ortodoxo español y, tras su exilio, uno de los pilares sobre los que se ha asentado la enorme expansión de esa doctrina en el mundo argentino, donde forma especialistas y crea una base institucional. Es autor de una amplia obra que incluye una interpretación de los sueños, así como versiones psicoanalíticas de trastornos psicosomáticos ${ }^{26}$.

Juan Comas Camps (Alayot, Menorca, 1900-México, 1979) fue inspector de primera enseñanza, y licenciado en ciencias naturales; pasó un tiempo en Ginebra, y publicó un volumen sobre tests; exiliado en México, se dedicó a la antropología, materia de la que logró obtener una cátedra en la UNAM y reconocimiento internacional ${ }^{27}$.

25 Herrero, F. (1997), «La escuela de Ginebra en la psicología aplicada española: La figura de Mercedes Rodrigo», Revista de Historia de la Psicología, 18 (1-2), pp. 139-150.

26 Zalbidea, M.A., CANTÓN, E. y CARPintero, H. (1991), «La influencia española en la psicología de los países latinoamericanos: Ángel Garma y el psicoanálisis en Argentina», Revista de Historia de la Psicología, 12(2), pp. 175-182; ZALBIDEA, M.A., CANTón, E. y CARPintero, H. (1992), «Un estudio sobre el impacto científico de Ángel Garma». Revista de Historia de la Psicología, 13 (2-3), pp. 233-246.

27 HeRnández MorA, J. (1979), «Juan Comas Camps: esquema de una vida», Revista Española de Antropología Americana, 9, pp. 17-24; VV.AA. (1982). 
Juan Roura Parella (Gerona,1897- USA, 1983). Fue profesor de Escuela Normal, y luego de pedagogía en la Universidad de Barcelona. Se exilió a México, donde estudió en profundidad la obra de Spranger, y se interesó por el tema de la personalidad. Pasó luego a enseñar en la Wesleyan University, USA, y falleció en aquel país ${ }^{28}$.

José Peinado Altable (Valladolid, 1909-Benidorm, Alicante, 1995). Inspector de primera enseñanza, se formó en la Escuela Superior del Magisterio; en colaboración con su compañero Juan Jaén Sánchez (Guijo de Ávila, Salamanca, 1904-1990), publicaron trabajos sobre psicoanálisis y educación $\left(1932^{29}\right)$ y sobre paidología (1933), muy difundidos entre educadores antes de la guerra. Los dos ampliaron estudios en Ginebra, con Claparède y Piaget, y tras la guerra, su destino divergió. Peinado se exilió a México, donde se orientó hacia la psicología clínica, y enseñó en México y luego en Venezuela, regresando al cabo a España. Jaén, por su parte, permaneció como inspector en España, y publicó trabajos sobre psicología infantili ${ }^{30}$.

Finalmente, José Germain Cebrián (Málaga, 1897-Madrid, 1986), médico psiquiatra, dedicado a la psicología, colaboró con Lafora, y organizó con Emilio Mira la red de psicología aplicada creada en los años 30 en España. Amplió estudios en Cambridge con Bartlett. Tras la guerra, puso su esfuerzo en restablecer la continuidad de la psicología científica en nuestro país, dirigiendo un departamento de psicología experimental en el Consejo Superior de Investigaciones Científicas, donde se formaron y trabajaron los creadores de la psicología contemporánea (M. Yela, J.L. Pinillos, M. Siguán, F. Secadas...), y sentó las bases institucionales de la nueva disciplina ${ }^{31}$.

28 Collelldemont, E. i Vilanou, C., «En el centenari del naixement de Joan RouraParella (1897-1983)». En VV.AA (1997), Joan Roura Parella, Barcelona, Universitat de Barcelona.

29 JAÉn, J. y PeINAdO, J. (1932), Psicología pedagógica. Lo subconsciente y la educación, Madrid, Aguilar.

30 CARPintero, H., y Del Barrio, V. (1996), «La introducción de Piaget en España. La figura y obra de Juan Jaén», Revista de Historia de la Psicología, 17 (3-4), pp. 186-193; PELÁEz Reoyo, T. (1996), «José Peinado Altable (1909-1995): aportaciones a la psicología y educación españolas e iberoamericanas». Valladolid, Eds. Universidad de Valladolid; CARPINTERO, H. (1997), «José Peinado y la influencia de Piaget en España», Revista de Historia de la Psicología, 18 (1-2), pp. 75-86; VV.AA. (1982).

31 Carpintero, H. (2004); Martí, C., Tortosa, F. y Carpintero, H. (1989), «Germain, neuropsiquiatra», Revista de Historia de la Psicología, 10 (1-4), pp. 161-172. 
2) Los educadores interesados por la psicología.

El grueso de nombres de nuestra selección corresponde claramente a educadores. La razón es clara: junto al hecho de no haber psicólogos, había una considerable masa de maestros y profesores que habían asumido la idea de que la reforma y elevación del nivel educativo pasaba por introducir en la enseñanza una fundamentación científica, lo que implicaba conocer y aprovechar conocimientos propios de la nueva psicología. Ya en la Escuela Superior del Magisterio se introdujo la enseñanza de la paidología, especialidad en la que destacaron figuras como Rufino Blanco (1861-1936) y Domingo Barnés (1879-1940), y en la que se buscaba integrar la psicología y la biología infantiles. Con ello se abrió el camino para una formación en psicología evolutiva, tema básico que había de guiar la acción del educador.

Aunque hay un grupo considerable de nombres de los que carecemos de fecha de nacimiento, la distribución de los pensionados según un ritmo generacional, a partir del examen del grupo bien identificado, muestra la existencia de un pequeño núcleo que corresponde a figuras de la generación del 98 y, en cambio, una nutrida presencia de los de las dos generaciones siguientes: la de 1886 y la de 1901. No obstante, dada la gran cantidad de nombres de que nos faltan datos para situarlos generacionalmente, haremos su presentación atendiendo a otros rasgos de semejanza o afinidad.

Hay algunos nombres que representan una primera ola de pensionados, y que pertenecen a la generación del 98. Ateniéndonos a los nombres de cronología determinada, hay que mencionar a Luis de Zulueta Escolano (n.1878), Luis de Hoyos Sainz (n.1868) y Ángel Llorca García (n.1866). Los tres iban a actuar, al igual que otros nombres bien conocidos de nuestra cultura, como 'avalistas' de los solicitantes más jóvenes ${ }^{32}$. Los dos primeros fueron profesores en la Escuela Superior del Magisterio y luego de la Facultad de Filosofía y Letras de Madrid, y renovaron el clima intelectual con los cursos que allí impartieron. Zulueta (Barcelona, 1978-Nueva York, 1964) fue profesor de historia de la educación, aunque en los años de la república tuvo una apretada actividad política, y después se exiliaría. Conviene notar que el trabajo de Jaén y Peinado sobre el psicoanálisis en el ámbito educativo estaba inspirado por las clases que sobre ese tema dio Zulueta en los años 1929-193033. Hoyos (18681951), por su parte, fue catedrático de fisiología humana y de higiene escolar, y renovó y modernizó los estudios de antropología física y cultural. Y en lo

32 MARÍN, T. (1990).

33 Carpintero, H. y Mestre, M.V. (1984), Freud en España, Valencia, Promolibro. 
que respecta a Ángel Llorca (Alicante, 1866- Madrid 1942), es notorio su liderazgo en el cuerpo del Magisterio. Fue maestro, formado en el entorno de M.B. Cossío y de L. Simarro; ocupó la dirección del Grupo Escolar «Cervantes», de Madrid impulsando la nueva pedagogía, y tuvo especial proximidad con el Instituto Juan Jacobo Rousseau de Ginebra. Alrededor de su figura se sitúan pensionados más jóvenes, que son educadores vinculados al mismo Grupo, como Justa Freire Méndez y Elisa López Velasco, ambas recordadas como especialistas en el campo del dibujo en la escuela, o también Manuel Alonso Zapata (Yegen, Granada, 1893-1936), activo promotor de la Escuela Nueva. Otros directores de grupo escolar que también tuvieron ese interés por la psicología parecen ser Virgilio Hueso y África Ramírez de Arellano, ésta especialmente interesada en la pedagogía de Decroly.

Hay por otra parte un interesante grupo formado por educadores que ocupan lugares muy destacados en el mundo de la organización y la política educativa de la II República. Aquí hay que incluir a Fernando de los Ríos Urruti (Ronda, 1978-Nueva York, 1949), ministro de Instrucción Pública y embajador de la República - lo consideramos luego, entre los especialistas en derecho-; los inspectores generales Antonio Ballesteros Usano (Córdoba, 1896México, 1974) y Fernando Sainz Ruiz (Granada, 1891-Chicago, 1957), María de Maeztu Whitney (Vitoria, 1882-Buenos Aires, 1947), profesora en el Instituto Escuela y directora de la Residencia de Señoritas; Pedro Roselló Blanch (Gerona, 1898-Ginebra, 1970), pedagogo que mantuvo estrecha relación con Piaget y fue director adjunto del Bureau International d'Éducation, y Francisca Bohígas Gavilanes (Barcelona, 1898-1873), profesora que fue diputada por la CEDA en 1933, que tuvo intervenciones sobre temas educativos, y luego ocupó puestos destacados en la posguerra. A excepción de esta última, todos los demás terminarían su vida en el exilio.

Hay un núcleo de profesores de Escuela Normal o inspectores de primera enseñanza, casi todos formados en la Escuela Superior del Magisterio, que recibieron sin duda el estímulo de las enseñanzas paidológicas y se interesaron por la nueva psicología, incluso cuando luego derivaran hacia otros estudios o especialización. Anotemos aquí a Pablo (Pau) Vila Dinarés (Sabadell, 1881-Barcelona, 1980), profesor de geografía de escuela Normal, luego exiliado, y autor de destacados estudios sobre geografía de Colombia y Venezuela; Carmen Abela y Espinosa de los Monteros (n. Chiclana, 1875), maestra un tiempo en Huete (Cuenca), y pensionada en los años 20; Salvador Ferrer $\mathrm{Cu}-$ lubret, (Gerona, 1902-1985), autor de una monografía sobre la Escuela Superior (1973); Juana Moreno de Sosa, profesora del Instituto Escuela; Julia Morros Sardá (n. León, 1902) profesora de ciencias; Gervasio Manrique 
Hernández (Soria, 1890-Madrid, 1978), inspector muy interesado en los problemas de la orientación escolar y profesional; Juana Ontañón Valiente (Madrid, 1886-México, 1972), profesora de literatura; aquí hallamos también a Antonio Gil Muñiz (Ceuta, 1892-Málaga, 1965), profesor de Escuela Normal, varios años separado de la cátedra, autor en colaboración con V. Pertusa de una Pedagogía moderna (Pertusa y Gil, 1922) que estaría basada en estudios empíricos sobre la psicología de los escolares madrileños, como soporte de una "pedagogía netamente española»"34; José Plata Gutiérrez (Mengíbar, 1904-Madrid, 1984), formado en la Escuela Superior, especialista en la educación de ciegos; Ramón Carrera, Concepción Majano (n. Toledo, 1891), Víctor Masriera Vila (n. 1875), Luis Leal Crespo, Jesús Sanz Poch, Mariano Sáez Morilla, Isidoro Boix Chaler, Federico Doreste Betancor (n. Lanzarote, 1886), Ricardo Torroja Valls (Barcelona, 1894-Venezuela, 1960)...

Muchos maestros tuvieron oportunidad de tener un contacto con la psicología a través de viajes de estudio. Parecen haber tenido una estancia ya duradera, propia de pensionados, Isidoro Boix, Dionisio Prieto Fernández o Tomás de Santiago González, entre otros.

Hay otro núcleo que claramente gira en torno a la pedagogía de niños con deficiencias sensoriales o mentales. Recuérdese que desde finales del XIX se había ido desarrollando en Europa una pedagogía terapéutica -E. Séguin, M. Montessori...-, y en 1910 se creó el Patronato de Sordomudos, Ciegos y Anormales; poco después, en 1914 se constituye el Patronato Nacional de Anormales, donde por un corto tiempo trabajaron N. Achúcarro y G. Rodríguez Lafora. Entre los pensionados de nuestro estudio se hallan, junto a G.R. Lafora, Jacobo Orellana Garrido — que fue director del Colegio Nacional de Sordomudos, desde donde impulsó el estudio y la atención terapéutica a ese problema ${ }^{35}$ - a María Luisa Navarro, — esposa de Lorenzo Luzuriaga, con quien irá al exilio tras la guerra-, Carmen Abela, Carmen Gayarre Galbete (1900-1996), Dolores González Blanco (n. Cuenca, 1892), Francisco García Almería, Adelina Méndez de la Torre, Elvira Ortega Pérez, Josefa Plaza Arroyo, Victoria Díaz Riva (Lugo, 1904-Málaga, 1972), Mariano Nuviola Falcón - pedagogía de ciegos - y María Soriano Llorente (n. Valencia, 1900), entre otros. Esta última fue profesora de la Escuela Central de Anormales (1922),

34 SÁnchez Sarto, L. (dir.) (1936), Diccionario dc Pedagogía (2 vols.). Barcelona, Labor, vol. I, p. 167.

35 ORellanA, J., «La enseñanza de los sordomudos», en VV.AA. (1936), Libro guía del maestro, Madrid, Espasa Calpe; Rodríguez Lafora, G., «La enseñanza de los anormales mentales». En VV.AA. (1936). 
aneja al Instituto Nacional de Sordomudos y Ciegos, centro que en 1960 se convertiría en Instituto de Pedagogía Terapéutica; ella misma encabezó el movimiento de pedagogía terapéutica en España tras la guerra civilib.

Hubo también algunos pensionados cuyo interés por la psicología fue unido al que sentían por otras especialidades muy concretas: las matemáticas -Antonio Llorens Clariana-, la grafología - Matilde Ras Fernández (Tarragona, 1881-Madrid, 1969), figura destacada de esa especialidad-, el cine escolar — Lorenzo de la Peña Lobón—, la música —-Emilio Gazapo Abelló-, la gimnasia rítmica —Ángeles Martínez Suárez-, o la alimentación e higiene escolar -Dolores Nogués Sardá.

\section{Otros pensionados.}

También se interesaron por la psicología pensionados procedentes de otras facultades y que luego atenderían a otros problemas propios de su especialización.

Hallamos aquí un pequeño núcleo de juristas, en el que se incluyen las figuras de Fernando de los Ríos, catedrático de derecho político vinculado a la ILE al que ya hemos mencionado, quien enseñó en la New School for Social Research, de Nueva York, durante su exilio; Eugenio Cuello Calón (Salamanca, 1879-Santander, 1963), figura notable del derecho penal y la criminología, que sin embargo hizo su memoria sobre los procedimientos experimentales para el estudio de niños anormales (Marín, 1990, 192); Luis Jiménez de Asúa (Madrid, 1889-Buenos Aires, 1970), figura internacionalmente conocida en derecho penal, con puestos de gran responsabilidad durante la república, y destacado introductor de ideas psicoanalíticas en las concepciones penales. También interesa la criminología a Jaime Masaveu, colaborador de Q. Saldaña. Se ha de contar aquí además con Blas Ramos Sobrino (1891-1955), especialista en filosofía del derecho, campo que también cultiva con gran éxito Luis Recasens Siches (Guatemala, 1903-México, 1977), pensador próximo a Ortega y cultivador también de la sociología, con gran actividad en México, a donde se exilió tras la guerra.

Hay, por otro lado, un grupo también reducido pero interesante de médicos: Santos Rubiano Herrera (Sevilla, 1871-1930), figura notable de la medicina militar; Jimena Fernández de la Vega (Vegadeo, 1895-Santiago de Com-

36 Parajón, L., Del Barrio, V. y Herrero, F. (1996), «La aportación de Maria Soriano a la Educación especial en España», Revista de Historia de la Psicología, 17 (1-2), pp. 203-212. 
postela, 1984), una de las primeras especialistas en temas de genética; Miguel Prados Such (Málaga, 1894-Montreal, 1969), psiquiatra y psicoanalista, exiliado, profesor en Canadá; Manuel Peraita, (1908-1950), neurólogo; y Sisinio Álvarez Soriano (n. 1892), médico escolar.

Finalmente, situamos aquí a algunas otras figuras de nuestro mundo académico. Hay dos catedráticos universitarios de filosofía, que son figuras notables de nuestra cultura: Manuel García Morente (Jaén, 1886-Madrid, 1942), catedrático de ética en la Universidad de Madrid, figura próxima a Ortega y decano de la Facultad de Filosofía y Letras en los años de la república; y Joaquín Xirau Palau (Figueras, 1895-México, 1946), catedrático de lógica en la Universidad de Barcelona, figura ligada a la ILE - es autor de un estudio sobre M.B. Cossío - y colaborador con Mira y López en el Instituto Psicotécnico de la Generalitat de Cataluña, antes de la guerra; exiliado en México, y profesor allí de filosofía en la universidad ${ }^{37}$. También situamos aquí a Eugenio Montes Domínguez (Vigo, 1900-Madrid, 1982), catedrático de literatura de instituto, fundador de Falange Española con J.A. Primo de Rivera y escritor notable, miembro en su día de la Real Academia Española.

Catedráticos de instituto fueron, además de los citados en el apartado 'psicólogos' por su mayor concentración en este campo, Pedro Guirao Gabriel, y Joaquín Álvarez Pastor (1885-1950), que fue filósofo y educador, director del instituto Luis Vives de Valencia, y ocupó puestos de algún relieve, como consejero de Instrucción Publica antes de la guerra; se exilió a México en 1939, y fue profesor de lógica en la Facultad de Filosofía y Letras de la UNAM; había tenido pensiones de estudio para Alemania y Francia.

\section{LOS CENTROS DE INTERÉS DE LOS PSICÓLOGOS}

El examen de los centros de investigación que acogieron a estos pensionados refleja bien las tendencias científicas y los intereses preeminentes en nuestro país, situado por aquellos años en un marco esencialmente europeo, donde dominaba una psicología funcionalista, más orientada hacia la mente humana y los procesos aplicados que hacia la entonces naciente psicología de la conducta norteamericana.

Fueron muy diversos los centros de investigación, laboratorios, universidades, visitados en los distintos países.

37 Siguán, M. (1995), Centenari Joaquim Xirau (1895-1995), Barcelona, Departament de Cultura, Generalitat de Catalunya. 
Por lo que se refiere a los Estados Unidos, el centro más visitado era el Teachers College de la Universidad de Columbia, en Nueva York, centro de estudios graduados de educación, donde John Dewey enseñó (entre 1904 y 1929) Psicología, Ética social, Educación de anormales y otras materias afines.

En cuanto a Inglaterra, algunos se aproximaron al Laboratorio Central de los Hospitales Mentales de Londres, donde trabajaba Frederick Mott (Miguel Prados Such); otros trabajaron en el Laboratorio de Psicología Experimental de la Universidad de Cambridge, con figuras como Myers y Bartlett (tal es el caso de Rodrigo Lavín y Germain), que desarrollaban una visión funcionalista, y estudiaban una psicología humana próxima a la vida, un tanto al margen de las polémicas entre escuelas que eran habituales aquellos días. Hubo también muchos pensionados que, procedentes del área psicopedagógica, se limitaron a visitar diferentes escuelas normales o asistieron a congresos pedagógicos.

En Alemania, la psicología mantuvo hasta los años 30 una situación de gran actividad y de muy alta calidad, como ha quedado reflejado en el informe de Viqueira antes mencionado. La Universidad de Berlín, con Stumpf, Spranger, Köhler, Wertheimer, Vogt, etc. recibió a algunos pensionados para realizar sus estudios de psicología y psiquiatría (Rodríguez Lafora, Viqueira, Moreno). Algunos otros viajaron a Leipzig, donde permaneció Wundt hasta 1917, junto a figuras como Rupp, Dessoir o Klemm; otros prefirieron trabajar en el Instituto de Pedagogía y Psicología experimental, que creó la Sociedad de Maestros de Leipzig en 1906 para fomentar la formación científica de estos. En la Universidad de Marburgo se podía estudiar Psicología y Psicopedagogía con Natorp, Hartmann, Cohen, etc, gracias a la existencia del fuerte grupo de pensadores de orientación neokantiana citados (García Morente, M. de Maeztu...).

El centro más relevante para la psicología de los visitados en Austria fue el Instituto de Psicología de Viena, fundado en 1922, donde trabajaban Charlotte y Karl Bühler - su director-, H. Hetzer, L. Schenck y E. Brunswick, entre otros (C. Gayarre...). Este se convirtió en uno de los primeros centros dedicados al estudio de la psicología del niño, y varias obras de sus directores vieron por esos años la luz en castellano.

Instituciones frecuentadas por estos pensionados en Francia fueron el Instituto de Psicología y Pedagogía de la Sorbona, organizado en 1920 (Zulueta, Masriera, Morros...), que reunía a figuras como Piéron, Janet, Dumas, Wallon...; el Laboratorio de Psicotecnia de la «École pratique des Hautes Études» (1908), donde trabajará Piéron, con Lahy, y la dirección del Dr. Toulouse, (Hueso, del Peso...); el Instituto Nacional de Sordomudos y Ciegos (Orellana, 
M. Cutanda...) y el Instituto Nacional de Orientación Profesional de París, creado por H. Piéron en 1928, para fomentar la psicología aplicada ${ }^{38}$.

Bélgica fue paso casi obligado para los interesados en la clínica y la pedagogía de la deficiencia mental. Desde 1897, las autoridades de Bruselas se preocuparon por fomentar la enseñanza especializada para niños deficientes mentales, retrasados o con algún tipo de anormalidad. La labor desarrollada allí por Ovide Decroly, creador de la «École de l'Ermitage» (1907), junto con sus colaboradores, alcanzó renombre internacional. Muchos educadores interesados en la Psicología infantil escogían como institución de destino el Instituto Decroly (Herrero García, Doreste, Abela, Almazán, etc.), o el Instituto de Psicopedagogía de la Universidad de Bruselas, donde impartían clases Decroly, Vermeylen, o Johnkheree (Ballesteros, Alfaya, Sisinio Álvarez, Doreste...). Algunos estudiaron en el Instituto Nacional de Sordomudos y Ciegos de la misma ciudad, otros en la Universidad de Lovaina y su Laboratorio de Psicología Experimental, con Michotte, Thierry, etc.

\section{El CASO DE LA ESCUELA DE GINEBRA}

En cuanto a Suiza, aunque algunos pensionados realizaron estudios oficiales de Psicología en la Universidad de Ginebra (M. Rodrigo, P. Roselló), la institución más visitada es sin duda el «Institut Jean Jacques Rousseau», objeto de una buena parte de las pensiones concedidas en psicología ${ }^{39}$. La relevancia de estas pensiones para la psicología española merece que nos detengamos en este centro un poco más, pues la llamada «Escuela de Ginebra» de psicología iba a ejercer un papel fundamental en el desarrollo de la psicopedagogía y de la psicotecnia españolas. Sus principios y creaciones fueron asumidos enseguida en nuestro país como las herramientas adecuadas para llevar a cabo el plan de reformas educativas y sociales que las instituciones demandaban ${ }^{40}$.

El núcleo inicial de la «Escuela de Ginebra» era, efectivamente, el Instituto J.J. Rousseau, fundado en 1912 en esta ciudad por el médico Edouard Claparède (1873-1940) y el educador Pierre Bovet (1878-1965). Allí se incorpo-

38 NiCOlas, S. (2002), Histoire de la psychologie française: Naissance d'une nouvelle science, Paris, InPress.

39 Herrero, F., García, E., y CArpintero, H. (1995).

40 LAfUente, E., y FerRÁndiZ, A. (1997), «La recepción de Claparède en España (19001936)», Revista de Historia de la Psicología, 18(1-2), pp. 151-165. 
raría, en 1921, la gran figura de Jean Piaget (1896-1980). No sólo fue un centro investigador, sino también un aglutinador de las principales figuras de la psicopedagogía europea, y lugar de difusión de las nuevas ideas y aplicaciones científicas de la psicología infantil y orientación profesional nacidas en el seno de su Escuela ${ }^{41}$.

Con una orientación funcionalista, que ponía el acento en las capacidades e intereses del niño como base de su educación («discat a puero magister», esto es, 'aprenda el maestro del niño', era su lema), promovieron la enseñanza activa basada siempre en un conocimiento científico, experimental en lo posible, de la mente infantil ${ }^{42}$.

Claparède gozaba de un gran prestigio internacional. No sólo impulsó el estudio científico de la psicología infantil, sino que promovió el desarrollo y organización de la psicotecnia y la psicología aplicada, patrocinando la creación de la Sociedad Internacional de Psicotecnia, en 192043. También tuvo desde muy pronto gran influencia en la psicología española Jean Piaget, colaborador de Claparède en sus primeros años ginebrinos, que ha llegado a ser uno de los más creativos e influyentes psicólogos de nuestro siglo. Los libros de estos autores fueron de consulta habitual entre los educadores y 'psicólogos' españoles, en algún caso traducidos y publicados en español antes de su aparición en francés.

La estrecha relación de algunos educadores españoles con los maestros de Ginebra facilitó la difusión de sus ideas entre el magisterio de los años $20 \mathrm{y}$ 30. Mercedes Rodrigo, Juan Jaén, José Peinado, Pedro Roselló, José Germain entre otros, se familiarizaron con las ideas y con las técnicas de investigación utilizadas en Ginebra, y crearon lazos que se mantuvieron a lo largo de los años, incluso después de extinguida la JAE, ya desde el exilio. Esa proximidad y familiaridad se reavivó, después de la guerra, gracias al esfuerzo de Germain, y más tarde, del grupo de Barcelona liderado por M. Siguán, que fortalecería sus contactos con Piaget y alentaría la línea piagetiana de estudios psicológicos en nuestro país.

Incluso se creó una Asociación Española de Antiguos Alumnos y Amigos del Instituto Rousseau, que fomentó los contactos con aquel centro; así, por su mediación, Claparède visitó Madrid en 1923 para dar una serie de conferencias en el Museo Pedagógico y en la Junta de Pensiones para Ingenieros y

41 Carpintero, H., y Herrero, F. (2002), «Early Applied Psychology (History of IAAP)». European Psychologist, 7(1), pp. 39-52.

42 Bovet, P. (1934), La obra del Instituto J.J. Rousseau, Madrid, Espasa Calpe.

43 LAfuente, E., y Ferrándiz, A. (1997). 
Obreros $^{44}$; también Claparède patrocinó la realización de reuniones internacionales de la Sociedad Internacional de Psicotecnia en Barcelona, cuyo Instituto de Orientación Profesional apreciaba grandemente. Muchos años más tarde, la Universidad de Barcelona concedió a Piaget su doctorado Honoris Causa, reafirmando así la larga relación que desde los años 20 había aquel mantenido con nuestros educadores y psicólogos.

Ginebra, en suma, fue la puerta principal por donde la psicología española especializada en temas infantiles y aplicados mantuvo una estrecha conexión con la psicología internacional. Representa, sin duda, un ejemplo y un modelo de logro de los objetivos científicos y organizativos que animaron a la JAE en los años de su existencia.

\section{UN PROYECTO DE PROGRESO PARA LA PSICOLOGÍA}

Entre los distintos documentos aportados por los psicólogos como resultado de sus estudios en el extranjero, destaca una memoria que presentó en 1915 Juan Vicente Viqueira sobre la enseñanza de la psicología en las universidades alemanas. Al parecer, con ocasión de una primera beca para Berlín, había antes remitido un breve trabajo sobre «Las direcciones actuales en la psicología», que apareció publicado en el BILE en $1914^{45}$, donde resume un trabajo del psicólogo alemán Stumpf de modo bastante esquemático. En cambio, este es un documento que presenta con sencillez y precisión el estado real de la psicología alemana, y singularmente la experimental, que «es la reinante hoy día» ${ }^{46}$. En él se recogen unas esenciales diferencias entre la situación española y la alemana, y se hace una propuesta que hubiera facilitado el desarrollo científico.

En efecto, para su autor, la distancia intelectual en ese campo científico entre nuestros dos países es tan grande, que apenas cabe la comunicación. Por eso afirma que es necesario «crear ... un pequeño y modesto Instituto de Psicología ${ }^{47}$ que haga posible y fecunda la colaboración. Tal sugerencia habría hecho avanzar varias décadas nuestra psicología.

\footnotetext{
44 Marín, T. (1988), pp. 230 y 231; LAfuente, E. y Ferrándiz, A. (1997), p. 156.

45 ViQueIRA, J.V. (1914), «Las direcciones actuales en la psicología», BILE, 647, pp. 6263 ; vid. BLANCO, F. (1996).

46 ViQueirA, J.V. (1915). «La enseñanza de la psicología en las universidades alemanas». Madrid, Separata de Anales de la Junta para Ampliación de Estudios e Investigaciones Cientificas, T. 16, memoria $1^{\text {a }}$, p. 9.

47 ViQUeIRA (1915), p. 8.
} 
Hace notar que la investigación científica no es erudición, sino inquisición de la verdad a partir del conocimiento de los fenómenos. Por eso en ella es esencial el método: así afirma que «todo resultado de la ciencia carece de valor y es incomprensible sin el conocimiento del método» ${ }^{48}$. Como no hay en España ni personal preparado, ni lugares donde trabajar o hacerlo luego al volver, si no se cambia la actitud y preparación de los que desean cultivar esa ciencia, los esfuerzos de la JAE vendrían a resultar «estériles»"49.

Viqueira analiza los rasgos de la universidad alemana, que explican la situación de la ciencia en ese país. Aquella busca «formar...especialistas» ${ }^{50}, \mathrm{y}$ va guiada por «la supremacía del saber teórico». Y aunque hay un gran número de personas interesadas en la psicología aplicada, - tendencia que ve influida por la manera de pensar americana - la universidad alemana considera lo aplicado como secundario, y busca «alejar» todo ese mundo de la atención a las aplicaciones ${ }^{51}$.

En su descripción de la situación de hecho, menciona los institutos de psicología ya existentes en varias universidades, subraya la limitación de sus recursos, y dice: «el personal no es tan abundante ni los medios materiales tan ricos como generalmente se supone» ${ }^{52}$; así, en el caso de Gottinga, todo el trabajo parece que han de hacerlo sólo dos personas: el investigador, G.E. Müller, y su ayudante ${ }^{53}$.

Atiende también al tema del plan de estudios, y anota la existencia de un curso general de psicología, dos cursos de introducción al trabajo de laboratorio, y un 'coloquio psicológico' donde se revisa la nueva literatura. Además, en ciertos lugares hay también cursos especiales sobre temas más monográficos: psicología infantil, aplicada, educativa... Y con ello, el estudiante pasa a investigar en un dominio determinado.

En cuanto al nivel de formación de esos alumnos, lo encuentra muy por encima del de los españoles: «la cultura general de cualquiera de los estudiantes - proceda del tipo que sea de segunda enseñanza...- es mayor que la de cualquier licenciado español» ${ }^{54}$. Y anota además que, contra lo que se suele pensar en España — donde la psicología sólo puede dar «trabajos litera-

\footnotetext{
48 ViQUEIRA (1915), p. 7.

49 ViQUEIRA (1915), p. 8.

50 VIQUEIRA (1915), p. 9.

51 VIQUEIRA (1915), p. 10.

52 VIQUEIRA (1915), p. 15.

53 VIQUEIRA (1915), p. 15.

54 VIQUEIRA (1915), p. 23.
} 
rios» ${ }^{55}$ - , la formación del psicólogo actual debe incluir ciencias: matemáticas, física y fisiología. Lo justifica dando cuenta de la moderna concepción de la psicología, que si bien es «ciencia de la conciencia»" ${ }^{56}$, es hoy un saber no introspectivo sino experimental, como una verdadera «ciencia natural», que busca aclarar la relación de los fenómenos mentales con la naturaleza - así, en la psicofísica. Es una tesis aceptada incluso por Husserl, cuyo trabajo sobre «La filosofía como ciencia rigurosa» cita. Junto a ello, está el hecho de que se mantiene un «íntimo contacto de la Psicología con la Filosofía», y eso se pierde cuando se sitúa la psicología en la facultad de ciencias, como ocurre en España, donde aquel contacto se desvanece ${ }^{57}$.

El trabajo termina recogiendo la polémica de aquella hora en Alemania, respecto a la necesidad o no de separar las cátedras de filosofía y las de psicología, y recuerda que, frente a Külpe y otros, Wundt ha solicitado el mantenimiento de aquella estrecha relación ${ }^{58}$. El problema, muy importante para la posición de la psicología en el marco universitario, fue bien detectado por Viqueira, evidenciando así su sensibilidad por la situación histórica y científica de esta ciencia en la Alemania del momento.

Este informe deja ver con claridad los problemas estructurales de la psicología, y los requisitos que habría que cumplir para hacer avanzar la investigación rápidamente y situarla en un nivel europeo, algo de que nuestra ciencia aquí carecía. Resituaba, además, la ciencia psicológica en un contexto de ciencia natural, y la acercaba a las ciencias naturales y a la matemática, lo que hubiera representado una positiva mejora de la formación de los investigadores interesados por estos temas. El informe no logró que se atendiera a sus propuestas. Pero sí hace ver con claridad la renovación mental que la JAE había hecho posible entre los nuevos pensionados, y su positivo influjo en la modernización del país.

\section{CONCLUSIONES}

Como puede verse por todo lo anterior, el elevado número de pensiones que la Junta concedía para realizar estudios psicopedagógicos y médicos en los

55 VIQUEIRA (1915), p. 26.

56 VIQUEIRA (1915), p. 23.

57 VIQUEIRA (1915), p. 26.

58 vid. ASH, M., «Wilhelm Wundt and Oswald Külpe on the institutional status of Psychology: An academic controversy in historical context». En BRINGMANN, W. \& TwENEY, R. (1980), Wundt studies, Toronto, Hogrefe, pp. 396-421. 
centros europeos más relevantes hace patente su interés y esfuerzo por la modernización educativa y el desarrollo científico y cultural en nuestro país, labor que se vio interrumpida, lamentablemente, por la Guerra Civil Española.

Es difícil evaluar en toda su importancia los resultados de esta política institucional. La fuerte conexión con la Escuela de Ginebra, mediante la comunicación con el Instituto Rousseau y la Universidad de Ginebra, la importante influencia de las ideas psicopedagógicas de Bühler, Decroly y de los principios de la «escuela nueva» europea, contribuyeron a crear una nueva mentalidad en los educadores españoles. La educación era entendida más como un proceso global de «formación» del educando que como mera «información». Se probaron y adoptaron nuevos métodos pedagógicos, se incorporaron a la enseñanza nuevas disciplinas, poniéndose especial énfasis en las lenguas modernas y en el principio de «actividad» en el proceso de aprendizaje, insistiendo en la creación de laboratorios escolares, etc., frente a la clásica exposición magistral. El Instituto-Escuela fue un intento de aproximación a un nuevo modelo de educación, basado en la coeducación, la búsqueda y la práctica de nuevas fórmulas de didáctica y evaluación escolar, y el laicismo, necesario para la formación de una mentalidad abierta y tolerante en los alumnos.

Los logros de la JAE quizá no alcanzaron todas y cada una de las metas propuestas, pero superaron multitud de obstáculos ocasionados por los conflictos políticos de la época, y desde luego redujeron notablemente el desnivel científico que España venía arrastrando secularmente. La Guerra supuso la radical paralización de toda la labor que se había llevado a cabo en pro del desarrollo de la ciencia y la cultura españolas. Por ejemplo, en el campo de la educación y la psicología se asistió a una vuelta al viejo modelo neoescolástico; y en la neuropsiquiatría se cortó la estrecha vinculación con el desarrollo de la disciplina, entrando en una etapa de aislamiento. Con todo, la impronta que la Junta dejó fue suficientemente profunda para mantener un impulso latente hacia el conocimiento, la investigación y la renovación, y para hacer posible la labor de muchas personalidades que, muchas veces sin apoyo ni recursos oficiales, en unos casos dentro, en otros fuera de España, fueron capaces de mantener vivos y de consolidar estos valores. Sobre estos esfuerzos e intentos, en mayor o menor medida, se asienta la cultura y la ciencia españolas de hoy. 


\section{ANEXO I: PENSIONADOS CON MATERIAS DE PSICOLOGÍA}

Abela y Espinosa de los Monteros, María del Carmen (Chiclana, Cádiz, 1875-?). Maestra en Huete. Esp. Educación Especial. (2, 8, 11).

Alonso Zapata, Manuel (Yegen, Granada, 1893- 1936). Magisterio, Grupo Esc. Cervantes, Dir. Grupo Esc. Salmerón, UGT. (1)

Álvarez Pastor, Joaquín (1885-1950). Catedrático de Filosofía en Málaga y León. Dir. Ins. Ens. Med. Luis Vives. Exiliado en México. (1)

Álvarez Soriano, Sisinio (1892-?). Maestro y Médico, Inspector médico escolar. Esp. Higiene y psicopatología infantil. $(4,9)$

Ballesteros Usano, Antonio (Córdoba, 1896-México, 1974). Maestro Escuela Sup. Magisterio, Inspector gral. Prof. Escuela Normal en México. (5)

Besteiro Fernández, Julián (Madrid, 1870- Carmona, 1940). Catedrático de Lógica Univ. Madrid. PSOE. (1)

Bohígas Gavilanes, Francisca (Barcelona, 1898- 1973) Magisterio, Inspectora. Diputada en Cortes en 1933 por la CEDA. (5, 10)

Boix Chaler, Isidoro (?-?). Magisterio, Misiones Pedagógicas. Escuela Nueva (1)

Carrera Díaz, Ramón (?-?). Prof. Esc. Sup. Mag. (4, 8)

Chousa López, Camilo (?-?). Maestro en Sevilla. $(\mathbf{1}, \mathbf{1 3})$

Cuello Calón, Eugenio (Salamanca, 1879-Santander, 1963). Catedrático de Derecho Penal en Granada, Barcelona, Madrid. Esp. Criminología. (5, 10)

Comas Camps, Juan (Alayot, Menorca, 1900-México 1979). Inspector, exiliado en México. Espec. Antropología, investigador en la UNAM. (2, 9)

Córdoba Rodríguez, José (1884-1955). Psiquiatra, profesor en la Univ. de Barcelona, Presidente de la Soc. de Neurología y Psiquiatría de Barcelona, etc. (10)

Dalmau Gratacós, Federico (Bañolas, Gerona, 1874-Gerona, 1926). Catedrático Ins. Ens. Med. Lovaina, Psic. Experimental $(\mathbf{2}, \mathbf{1 0})$

Díaz Riva, María Victoria (Lugo,1904-Málaga, 1972). Licenciada en Filosofía y Letras, inspectora escolar y alumna de J. Orellana (Trastornos del lenguaje y Educ. Especial) $(4,8,9,11)$

Doreste Betancor, Federico (Haría, Lanzarote 1886-?). Maestro, Director de Grupo Escolar en Barcelona y Esc. Sup. Magisterio. Esp. método Decroly (1)

Durán Pulis, Fernando (?-?). (1, 2)

Fernández de la Vega y Lombán, Jimena (Vegadeo, 1895- Santiago de Compostela, 1984). Lic. en Medicina Santiago Compostela (alumna de Nóvoa). Espec. herencia (10)

Ferrer Culubret, Salvador (Gerona 1902-1985). Magisterio, inspección. Libro sobre Esc. Sup. Mag. (1909-32), referente histórico en el área $(\mathbf{2}, \mathbf{5}, \mathbf{8}, \mathbf{1 0})$ 
Freire Méndez, Justa (?-?). Prof. Dibujo Grupo Esc. Cervantes. Misiones pedagógicas. (1) García Almería, Francisco (?-?). Maestro en Oviedo. Espec. sordomudística (2, 8, 9)

García Morente, Manuel (Arjonilla, Jaen, 1886-Madrid, 1942). Catedrático de Filosofía Univ. Madrid y profesor en la ILE. (1)

García Romo, Antonio (?-?). Lic. en Filosofía y Letras. (7, 11)

Garma Zubizarreta, Ángel (Bilbao, 1904-Buenos Aires, 1993). Médico. Psicoanalista. Fundador de la Sociedad Psicológica Argentina. Espec. med. psicosomática. (11)

Gayarre Galbete, María del Carmen (1900-1996). Prof. Fac. Filosofía Univ. Madrid. Espec. Psicopatología infantil. $(2,4,5,8)$

Gazapo Abelló, Emilio (?-?). Maestro (música), Grupo Esc. Cervantes de Madrid. (1)

Germain Cebrián, José (Málaga, 1897-Madrid 1986). Psiquiatra, colaborador de Lafora. Archivos de Neurobiología. CSIC. Soc. Esp. Psicología. (10)

Gil Muñiz, Antonio (Ceuta, 1892-Málaga,1965). Catedrático Esc. Normal en Córdoba (depur. tras guerra). (9)

González Blanco, María de los Dolores (Cuenca,1892-?) Prof. Esc. Nor. de Cuenca. Esp. Educ. Especial. (1)

Guirao Gabriel, Pedro (?- ?). Catedrático Ins. Ens. Medias. (2)

Heredero Pérez, Manuel (?-?). Catedrático Ins. Ens. Medias. (2, 4, 12)

Herrero Bahillo, Fermín (Palencia,1871-Ávila, 1921). Dr. en Filosofía, Catedrático Ins. Ens. Medias en Ávila. Socialista. Espec. Psic. Cultural. $(12,13)$

Herrero García, Miguel (1895-1961). Catedrático de Literatura española Instit. Esc. S.E. (2)

Hoyos Sáinz, Luis de (1868-1951). Catedrático de Fisiol. Esc. Sup. Magisterio. Fund. Museo del Pueblo Español. Espec. Antropología. (2, 9, 10)

Huerta Naves, Ramón Luis (San Andrés, Asturias 1889-?) Maestro en Asturias y Madrid, exiliado en Francia durante 20 años. Presid. FETE. Espec. Higiene. (5, 11)

Hueso Moreno, Virgilio (?-?). Magisterio. Dir. Grupo Esc. La Florida, Madrid. Libros de texto. Espec. Psicopedagogía. (6, 9)

Jaén Sánchez, Juan (Guijo de Ávila, Salamanca, 1904-1990). Inspección, prof. Esc. Sup. Magisterio, discip. de Luis Zulueta. Esp. Psicología infantil. (5, 9)

Jiménez Asúa, Luis (Madrid 1889-Buenos Aires, 1970). Prof. Dcho. Penal Univ. de Madrid. Diputado por el PSOE en 1931. Diplomático, exiliado en Argentina y Pres. de la República en el exilio. (5)

Leal Crespo, Luis (?-?). Prof. Normal, exiliado en México. Esc. Nueva. (4)

Llorca García, Ángel (Orxeta, Alicante, 1866-1942). Magisterio. Dir. Grupo Esc. Cervantes. Discip. de Cossío y Simarro. (1)

Llorens Clariana, Antonio (?-?). Estudios de Psic. Pedag., Pedag. y Psic. Comparada en USA y Alem. $(3,4,5,9)$ 
López Velasco, Elisa (?-?). Prof. Dibujo Grupo Esc. Cervantes. Rel. con la ILE. UGT. (1)

Luis André, Eloy (Mourazos, Orense, 1876-Madrid, 1935). Catedrático Ins. Ens. Medias Cisneros (Madrid). Psicólogo wundtiano. (1)

Maeztu Witney, María de (Vitoria, 1882-Buenos Aires., 1947). Pedagoga. Discip. de Unamuno y Ortega. Dir. Residencia de Señoritas, Instituto-Escuela. Exiliada en Argentina. (9)

Majano Araque, Concepción (Villasequilla, Toledo, 1891-?). Prof. Normal en Madrid. Espec. Organiz. Esc. (9)

Manrique Hernández, Gervasio (Osona, Soria, 1890-Madrid, 1978). Inspección. Espec. Orientación Profesional y Etnología. $(4,5,12)$

Martínez Suárez, María de los Ángeles (?-?). Prof. Fund. Glez-Allende de Toro. Gimn. rítmica (2)

Masaveu Masaveu, Jaime (?-?). Dr. en Derecho. Dir. Lab. Criminología con Q. Saldaña (1933-6). Pres. de la R.A. de Doctores de España. Espec. Psic. Jurídica. (2, 10)

Masriera Vila, Víctor (1875-?). Pintor, Prof. Esc. Sup. Magisterio. Fund. Asoc. Amigos URSS. Espec. Ped. del dibujo. (5)

Méndez de la Torre, Adelina (?-?). Magisterio. Precursora de las ikastolas. Espec. Educ. Especial. (9)

Miaja Carnicero, Amalia (?-?). Prof. de ciencias Es. Sup. Maestras Guipúzcoa. (5, 11)

Mingarro Sanmartín, José (?-?). Dr. en Derecho, prof. Universidad de Madrid. Exiliado en Cuba y México. $(\mathbf{2}, \mathbf{1 0}, \mathbf{1 3})$

Molina Pastor, Vicente (?-?). (1)

Montes Domínguez, Eugenio (Vigo, 1900-Madrid, 1982). Dr. en Filosofía (con Ortega). Catedrático de Literatura Ins. Ens. Medias. RAE, fundador FET. (1, 13)

Moreno de Sosa, Juana (?-?). Profesora en el Instituto-Escuela, Psic. Exper. en París, Berlín y Ginebra $(2,3,4,6,7,9,10,11)$

Morros Sardá, Julia (León, 1902-?). Lic. en Cc. Naturales. Catedrática Esc. Normal de Madrid. Prof. en el Instituto Escuela. Espec. Psic. Experimental e Higiene. Col. de Luis Hoyos. Exiliada en Argentina. $(\mathbf{2}, \mathbf{3}, \mathbf{4}, \mathbf{5}, \mathbf{8}, \mathbf{1 0})$

Muñoz Gaspar, José (?-?). (2)

Muñoz Gaspar, Jesús (?-?). (2)

Navarro Margoti, María Luisa (?-?). Prof. Col. Nnal. de Sordomudos. Misiones Pedagógicas. Espec. Pedag. sordomudos, Logopedia. Exiliada en Argentina. (4, 5, 10)

Nogués Sardá, Dolores (?-?). Prof. en la Esc. Normal de Ávila. Misiones Pedagógicas. Esp. enseñaza doméstica. $(\mathbf{2}, \mathbf{9})$

Nóvoa Santos, Roberto (La Coruña, 1885-Santiago de Compostela, 1933). Catedrático de Patología en Santiago y Madrid. Diputado socialista en 1931. (4, 7)

Nuviola Falcón, Mariano (?-?). Prof. Col. Nnal. Sordom. y Ciegos de Madrid, reformador de su metodología (1914). (2) 
Ontañón Valiente, Juana (Madrid, 1886.- México,1972). Catedrática Esc. Normal de Leng. y Lit. Exiliada en México, prof. Colegio Luis Vives y Univ. Femenina de Méx. Fund. Ateneo Pablo Iglesias de Pamplona. (5)

Orellana Garrido, Jacobo (?-?). Prof. Esc. Nnal. Anormales. Dir. Col. Nac. Sordomudos. $(2,8)$

Ortega Pérez, Elvira (?-?). Prof. Esc. Normal de Sevilla. Espec. Educ. Especial. (1)

Peinado Altable, José (Valladolid, 1909-Benidorm, 1995). Inspección. Exiliado en México y Venezuela, Catedr. en la UNAM y en la Central de Venez. Espec. Psic. Infantil y Clínica. $(5,9)$

Peña Lobón, Lorenzo de la (?-?). Maestr Gr. Esc. Cervantes de Madrid. Organiz. y Cinematogr. escolar (1)

Peraita y Peraita, Manuel (1908-1950). Neurólogo, colab. de Negrín en la Fac. Med. de Madrid, de Sacristán y de López Ibor. $(4,10)$

Peso Sevillano, José del (?-?). Inspector en Lugo. Psic. Experimental. (2, 4, 10)

Plata Gutiérrez, José (Mengibar, 1904-Madrid, 1984) Maestro en Córdoba y Cuenca. Catedrático Esc. Sup. en Ávila, Inspector en Madrid. $(\mathbf{2}, 4,5,9,10,11,12)$

Plaza Arroyo, Josefa (?-?). Profesora en la Esc. Nnal. de Anormales y Col. de Sordom. y ciegos. $(5,8,11)$

Prados Such, Miguel (Málaga, 1894- Montreal, 1969). Médico, psicoanalista e histólogo. Trabajó en el Instituto Cajal. Exiliado en Canadá, prof. de Psiquiatría en Montreal. (1)

Prieto Fernández, Dionisio (?-?). Maestro en el Grupo Esc. Cervantes de Madrid. Esc. Nueva. (1)

Prieto Fernández, Ildefonso (?-?). Maestro en el Grupo Esc. Cervantes de Madrid. Esc. Nueva. (1)

Ramírez de Arellano y Ramírez, África (c.1892 Valladolid?- ?). Maestra Sup. Dir. Grupo Esc. Mnez Pelayo de Madrid. Co-dir. Residencia de Señoritas. Educ. Especial. (9)

Ramón y Cajal, Santiago (1852-1934). Histólogo, Premio Nobel 1906. (1)

Ramos Sobrino, Blas (Tiedra, Valladolid, 1891- 1955). Catedr. Filos. Derecho Univ. Granada, Sevilla, Valladolid y Madrid. Exiliado en México. $(\mathbf{1 , 6 , 1 3 )}$

Ras Fernández, Matilde (Tarragona, 1881-Madrid, 1969). Divulgadora de la grafología en España. Prof. en el Instit. Intern. de Boston en Madrid. (4, 8)

Recasens Siches, Luis (Guatemala, 1903- México, 1977). Catedrático de Filos. del Derecho en la Univ. de Madrid. Exiliado en México, impartirá clases en la UNAM. (1)

Ríos Urruti, Fernando de los (Ronda, 1878-N.Y., 1949). Catedrático de Derecho Político en Granada. Ministro socialista de Instrucción Pública en 1933. Exilio en USA. (5, 13)

Rodrigo Bellido, Mercedes (Madrid, 1891-Puerto Rico, 1982). Maestra. Psicotécnica en el Inst. Méd.-Pedag. de Lafora, y el Nnal de Or. Prof. Exiliada en Colombia y P. Rico $(2,4,5,6,9,11)$ 
Rodrigo Lavín, Cipriano (San Sebastián, 1881-Madrid, 1972). Dr. Medicina y CC. Nat. Ayte. de Simarro en la Universidad (Psic. Experim). Represaliado tras la guerra. (2)

Rodríguez Lafora, Gonzalo (Madrid, 1886-1971). Psiquiatra y neurólogo. Discip de Cajal y Simarro. Fund. Inst. Méd-Pedag. Pres. Cons. Sup. Psiquiátrico. Exiliado en México. $(7,11)$

Rodríguez, José César (?-?). Maestro del Grupo Esc. Cervantes de Madrid. Esc. Nueva. (1)

Roselló Blanch, Pedro (Calonge, Gerona, 1898-Ginebra, 1970). Inspección. Esc. Sup. Magisterio. Bureau Int. Educ. (Unesco). Colab. Piaget. $(\mathbf{2}, \mathbf{4}, \mathbf{5 ,} 6, \mathbf{9 , 1 1}, \mathbf{1 3})$

Roura Parella, Juan (Fortilla,1897- USA, 1983). Prof. Normal, Orient. Prof. Exiliado en México y USA. $(2,4,5,12)$

Rubiano Herrera, Santos (Sevilla,1871-1930). Médico militar. Veterano de Filipinas. Dir. Clin. Psiquiat. Militar de Ciempozuelos. (4)

Sáez Morilla, Mariano (Albacete_?-Navarra, 1937). Maestro y Lic. en Derecho. Director Esc. Normal en Navarra, Miembro de la Junta de protección de Menores. FETEUGT. (9)

Sáinz Amor y Alonso de Celada, Concepción (1897-1994). Dra. En Filosofía (Dir. Zaragüeta), Dir. Grupos Escolares, Prof. Pedag. Comprar. Univ. Barcelona. Espec. Rorschach. (8)

Sanz Romo, Marcelo (?-?). Espec. Educ. Física. (1, 10)

Sáinz Ruiz, Fernando (Granada, 1891-Chicago,1957). Magisterio. Insp. Gral. República. (2)

Sánchez Fuentes, Carmen (?-?). Maestra en la Esc. Normal de Madrid. (9)

Santiago González, Tomás de (?-?). Maestro del Grupo Esc. Cervantes de Madrid. Esc. Nueva. (1)

Sanz Poch, Jesús (?-?). Prof. Normal en Lérida, Pedag, Lit. y Bilingüismo. Esc. moderna. $(1,4,5,6,8,9,11)$

Serna y Espina, Luis de la (1907, Cabezón de la Sal, Santander -España, ?). Médico. Esp. Herencia (10)

Simarro, Luis (1851-1921). Primer Catedrático español de Psicología Experimental, Univ. de Madrid. (1)

Soriano Llorente, Maria (Valencia, 1900-Madrid, 1994?). Prof. Anormales, Titul. en el Inst. de O.P. de Madrid y en Logopedia en París. Dir. Esc. Central Anormales. $(5,8,9)$

Terrasa Pugés, Juan B. (?-?). Lic. en Filosofía. (1)

Torroja Valls, Raimundo (Barcelona, 1894 -Venezuela, 1960). Dtor. Esc. grad. aneja Normal de Barcelona. Educ. moral. Exiliado en Caracas. (1)

Vidal Roget, Augusto (Llagostera, 1909 - Barcelona, 1976). Maestro. Comunista, exiliado en USSR. Prof. Filol. Univ. de Moscú. Traductor. Psic. Educación. (9)

Vila Dinarés, Pablo (Sabadell, 1881-Barcelona, 1980). Catedrát. Geogr. Normal Barcelona. Exiliado en Colombia y Venezuela. $(\mathbf{2}, \mathbf{5}, \mathbf{9})$ 
Viqueira López, Vicente (Madrid, 1886-Bergondo, Coruña, 1924). ILE, Catedrático Ins. Ens. Medias. Espec. Psic. experim. (1, 2, 10)

Xirau Palau, Joaquín (Figueras, 1895-México, 1946) Catedrático de Filosofía en la Universidad de Salamanca y Barcelona. Exiliado en México. (1)

Zulueta Escolano, Luis (Barcelona, 1878-NY, 1964). Pedagogo de la ILE, Prof. Esc. Sup. Magist. Ministro de la II República. Exiliado en México. (11)

\section{LEYENDA DE LAS ÁREAS DE ESTUDIO DURANTE LAS PENSIONES:}

(1) Psicología general. (2) Psicología Experimental. (3) Psicología Animal. (4) Psicología Diferencial, Psicometría y Psicotecnia. (5) Psicología Evolutiva y Paidología.

(6) Psicología del Desarrollo Afectivo y Moral. (7) Psicología del Arte. (8) Psicolingüística. (9) Psicopedagogía. (10) Psicobiología y Psicofísica. (11) Psicopatología y Psicoanálisis. (12) Psicología del Trabajo. (13) Psicología social. 